\section{PSICOLOGIA IBEROAMERICANA}

\section{Psicología Iberoamericana}

ISSN: 1405-0943

psicología.iberoamericana@uia.mx

Universidad Iberoamericana, Ciudad de

México

México

Álvarez Ramírez, Leonardo Yovany; Cáceres Hernández, Laura

Resiliencia, Rendimiento Académico y Variables Sociodemográficas en Estudiantes Universitarios de

Bucaramanga (Colombia)

Psicología Iberoamericana, vol. 18, núm. 2, julio-diciembre, 2010, pp. 37-46

Universidad Iberoamericana, Ciudad de México

Distrito Federal, México

Disponible en: http://www.redalyc.org/articulo.oa?id=133915921005

- Cómo citar el artículo

Número completo

- Más información del artículo

Página de la revista en redalyc.org

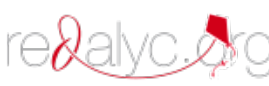

Sistema de Información Científica

Red de Revistas Científicas de América Latina, el Caribe, España y Portugal

Proyecto académico sin fines de lucro, desarrollado bajo la iniciativa de acceso abierto 


\title{
Resiliencia, Rendimiento Académico y Variables Sociodemográficas en Estudiantes Universitarios de Bucaramanga (Colombia)
}

\author{
Resilience, Academic Performance and Socio Demographic Variables into \\ a Group of University Students in the City of Bucaramanga (Colombia)
}

\author{
Leonardo Yovany Álvarez Ramírez \\ Laura Cáceres Hernández* \\ Universidad Autónoma de Bucaramanga, Floridablanca, Colombia
}

\section{RESUMEN}

El objetivo de este estudio fue explorar las dimensiones de la resiliencia, las correlaciones de ésta con la edad, el estrato socioeconómico, el número de hijos y las diferencias por género, estado civil y tipo de convivencia familiar en 180 estudiantes universitarios de ciudad de Bucaramanga. El estudio correlacional-transversal abordó una muestra no probabilística de estudiantes universitarios. Se utilizó el instrumento CRE-U (Peralta, Ramírez \& Castaño, 2006) para evaluar la resiliencia. Se hallaron factores resilientes en el grupo: Habilidades para solucionar problemas, humor y conductas vitales positivas. No se hallaron correlaciones significativas entre la resiliencia y la edad, el estrato socioeconómico ni el número de hijos. Las mujeres resultaron más resilientes que los hombres, así como los solteros y quienes viven junto a sus familias. La correlación entre la resiliencia y rendimiento académico fue débil. No se hallaron diferencias según el género ni el estado civil en la resiliencia de los examinados.

Descriptores: Universitarios, resiliencia, rendimiento académico y variables sociodemográficas.

\section{ABSTRACT}

The objective of this survey was to explore the dimensions of resilience, its correlations with age, economical status, the number of children and the differences by gender, civil status and sort of familiar convivence into a group of 180 university students in the city of Bucaramanga. The cross-correlational-transversal had a non probabilistic simple of university students he study used the CRE-U (Peralta, Ramirez, \& Castaño, 2006) to assess the resilience. Three resilience factors were found: Abbilities to solve problems, humor and positive vital behaviors. There was found insignificant correlations among resilience and age, economical status and number of children. Women seamed more resilient than men, so that single ones and those who live with their families. Correlation between resilience and academic performance was weak. There weren't found differences according gender and civil status in the resilience of examined ones.

Key words: university students, resilience, academic performance and socio demographic variables.

\footnotetext{
*Para correspondencia: Leonardo Yovany Álvarez Ramírez, Dirección: Programa de Psicología, UNAB. Calle 157 No.19-55. Cañaveral Parque. Florida blanca- Santander - Colombia, E-mail: leonardoalvarez64@gmail.com. Laura Cáceres Hernández, Dirección: Facultad de Psicología UNAB. Calle 157 No.19-55. Campus el Bosque. Florida blanca. Colombia. E-mail: lcaceres@unab.edu.co.
} 


\section{INTRODUCCIÓN}

La resiliencia se ha convertido en tópico de investigación debido a que está asociada a la salud mental y a los mecanismos de adaptación al entorno por parte del individuo. Asimismo, está relacionada con la confianza, el optimismo ante la adversidad porque permite al individuo reconocer sus propias posibilidades, confiar en la ayuda que pueda obtener de los demás y el manejo de las contingencias situacionales ante las cuales debe saber resistir y acometer, preservando la calidad de vida (Werner, 1989) teniendo en cuenta que las condiciones para la consecución de las metas no siempre son favorables y existen diferentes obstáculos que superan al individuo, ya sea en lo personal, lo interpersonal, el sistema y contexto propio en el cual el individuo se desarrolla y debe subsistir.

En el contexto educativo, la resiliencia juega un papel importante, ya que en éste, el individuo mide sus propias fuerzas frente a diferentes retos y demandas, no sólo de tipo académico sino psicosocial, sorteando situaciones exigentes que lo llevan a instancias dilemáticas en las cuales debe confrontarse a sí mismo para poder entender mejor su potencial y capacidades para fortalecerse, aprender y responder con eficacia, conservando su salud mental y su confianza en su potencial y habilidades. Las pocas investigaciones realizadas en torno al tema se han centrado principalmente en la identificación de características resilientes en población infantil (Bradley et al., 1994), en poblaciones víctimas de pobreza (Sammeroff \& Seiffer, 1992) y desastres, pero en población estudiantil ha sido muy poco explorada (Gargallo et al., 2002).

Este estudio pretende aportar al análisis de esta categoría psicológica en dicho contexto, relacionándola con aspectos del rendimiento académico así como otras variables sociales y demográficas de los estudiantes a fin de indagar por posibles relaciones que haya entre las dimensiones de la resiliencia y la edad, el nivel socioeconómico y las posibles diferencias de género, estado civil e integración con la familia. Estas exploraciones permiten revisar posibles conexiones que hasta el momento no se reportan claras entre la resiliencia y algunos factores sociodemográficos, tampoco, entre ésta y el desempeño académico y aprendizaje en los estudiantes universitarios.
El proceso de aprendizaje implica una gran dosis de motivación, lo cual supone no sólo resistir adecuadamente ritmos y exigencias de adaptación y respuestas de todo tipo, sino también la capacidad de autorregularse para responder en la medida adecuada sin caer en situaciones de exacerbación o alteración emocional, como indefensión, apatía, depresión, angustia. Algunos antecedentes acerca de la resiliencia en poblaciones estudiantiles revelan estas manifestaciones asociadas con la deficiencia de resiliencia (Bragagnolo et al., 2005), asimismo, las investigaciones de estrés en universitarios señalan que las carencias de autoconfianza crean un patrón de vulnerabilidad que deja a los estudiantes en condiciones de baja resistencia y escaso optimismo acerca de sus posibilidades y las del entorno para poder salir adelante, lo cual desencadena trastornos de diversa índole psicosocial, los cuales no siempre resultan atendidos por los servicios de apoyo institucionales debido a diversas razones, situación que incide en el desempeño académico, las relaciones sociales y la afectividad misma del estudiante (Solórzano \& Ramos, 2006).

Otras categorías psicológicas han sido exploradas en estudiantes que están relacionadas con la resiliencia, por ejemplo, factores como la ansiedad, rasgos de personalidad, auto concepto y asertividad; sin embargo, las dimensiones de la resiliencia, de manera específica no han sido objeto de investigación en la población universitaria (Bermúdez et al., 2006), lo cual no sólo tiene implicaciones para la investigación, sino educativas, prácticas y psicosociales, dado que la calidad de vida resulta esencial para desempeñarse adecuadamente en el contexto educativo y de hecho, ha de hacer parte de los objetivos educativos mismos (Garmezy,1994). Por estas razones, resulta importante emprender investigaciones dirigidas a identificar las dimensiones de la resiliencia y las relaciones que éstas pueden tener con otros aspectos de la vida del individuo y de su proceso de aprendizaje.

El presente estudio pretende evaluar las dimensiones de resiliencia en un grupo de universitarios y universitarias, y examinar las posibles correlaciones entre esta capacidad emocional y el rendimiento académico de la muestra estudiada; asimismo, evaluar otras posibles correlaciones con variables tales como la edad, el estrato socioeconómico y el número de hijos de los 
participantes. Finalmente, se examina si existen diferencias de género, estado civil y tipo de convivencia familiar en los examinados. Lo anterior, teniendo en cuenta que en lo hallado, sólo un estudio en Colombia aborda la relación entre la resiliencia y el rendimiento académico en estudiantes universitarios.

\section{MÉTODO}

\section{Diseño}

Se realiza un estudio no experimental, correlacionaltransversal, ya que no se manipulan las variables, sino que se busca establecer las posibles asociaciones existentes entre ellas, a saber: resiliencia, rendimiento académico y variables sociodemográficas (edad, género, estrato, número de hijos, convivencia familiar y estado civil) en estudiantes universitarios de la ciudad de Bucaramanga.

\section{Variables}

V1. Resiliencia. Capacidad del individuo para hacer introspección acerca de su vida y condición, de sus estados afectivos y de sus actos, de emprender acciones auto determinadas por su iniciativa, de mantener una actitud de humor genuina frente a diferentes circunstancias adversas o de pérdida afectiva, social o material, de interactuar de manera empática y asertiva construyendo vínculos sanos de soporte, protección e intercambio con independencia e interdependencia, moralidad y pensamiento crítico (Werner,1982; Wolín \& Wolín,1993).

V2.Rendimiento académico. Determinado por el promedio de calificación ponderado en una escala de 1 a 5 , en la cual 1 es el valor inferior, 5 el máximo y 3 el promedio de aprobación por asignatura según el sistema de evaluación universitario en Colombia.

V3.Demográficas. Estrato socio económico.

V4.Demográficas. Edad.

V5.Demográficas. Género.

V6.Demográficas. Número de hijos.

V7.Sociales. Convivencia familiar.

V8.Sociales. Estado civil.

\section{Hipótesis}

H1. Se encontrarán dimensiones de la resiliencia tales como el humor y la iniciativa en el grupo estudiado, dado que estas son dos componentes característicos en quien la posee.

H2. La correlación entre la resiliencia y el rendimiento académico de los estudiantes examinados mostrará valores positivos fuertes.

H3. La resiliencia en los estudiantes motivo de estudio incrementará cuando el estrato socioeconómico sea más bajo en los examinados.

H4. Las capacidades resilientes serán mayores en las participantes mujeres, en los de mayor edad y con mayor número de hijos, así como en los casados.

\section{Participantes}

El estudio incluyó una muestra por conveniencia de 180 estudiantes universitarios de quinto y sexto semestres de cuatro universidades públicas y privadas de la ciudad de Bucaramanga, 95 de género masculino y 85 femenino; los participantes a los cuales se tuvo el acceso tenían edades entre los 17 a 37 años, niveles socioeconómicos del tres al seis, cualquier estado civil, ocupación, con o sin hijos y vivían o no con sus familias. Se incluyeron estudiantes sin discriminar tipo de carrera ni tipo de universidad (pública o privada).

\section{Instrumentos}

El instrumento que se utilizó fue el Cuestionario de resiliencia para estudiantes universitarios (CRE-U) (Peralta, Ramírez \& Castaño, 2006). Esta herramienta posee una validez de constructo basada en el modelo de resiliencia de Emma Werner (1982) y Wolín \& Wolín (1993). El modelo define la resiliencia en función de factores personales que sirven de protección y fuerza a las personas en situaciones difíciles o adversas. Las subescalas del instrumento son: introspección, iniciativa, humor, interacción, independencia, creatividad, moralidad y pensamiento crítico. El instrumento tiene una validez de experto, ya que fue evaluado por tres investigadores en el tema; la confiabilidad se realizó por medio de una prueba piloto a 146 estudiantes de la Universidad del Norte (Colombia). La escala posee una confiabilidad alpha de Cronbach de 0.70, lo cual indica que ésta es media alta, suficiente para realizar una investigación.

El CRE-U tiene un total de 90 ítems, con cinco opciones de respuesta estilo escala Likert, en la cual 1 significa nunca, 2 casi nunca, 3 a veces, 4 casi nunca y 5 siempre. 
Los datos sociodemográficos se recogieron a través del diligenciamiento de una ficha en la cual se solicitaba incluir la edad, el género, el estado civil, el estrato socioeconómico, si se convivía o no con la familia, si se tenían hijos o no.

Los datos sobre el rendimiento académico se recogieron del promedio académico ponderado del estudiante hasta el semestre actual (quinto a sexto semestres), según su carrera y en la escala de calificación de 1 a 5, en que 1 es la calificación mínima y 5 la máxima del desempeño académico.

\section{Procedimiento}

Se contactó a los participantes en cuatro universidades de la ciudad de Bucaramanga a quienes se les aplicó el cuestionario de resiliencia para estudiantes universitarios CRE-U, previas indicaciones sobre diligenciamiento y explicación del consentimiento informado.

En una segunda fase se llevó a cabo la recopilación de datos en el programa Excel de cada una de las variables sociodemográficas, al igual que los datos referentes al rendimiento académico y de los 90 ítems de cada prueba, en matrices de datos. Se efectuó el análisis factorial, las correlaciones y la prueba $t$ de Student; además, la tabla de frecuencias de las variables para la caracterización de la muestra, lo anterior por medio del programa SPSS 12.0.

\section{Resultados}

En la Tabla 1 se muestran los resultados del análisis factorial con rotación Varimax aplicada a la muestra de estudiantes universitarios del presente estudio. Los resultados de la rotación para la muestra $\left(n_{1}=180\right)$ arrojan una solución de 29 componentes que explicaron el $70.544 \%$ de la varianza de la cual fueron seleccionados inicialmente nueve componentes que explican un $20.966 \%$ de la varianza. (Para esta selección se tuvieron en cuenta sólo aquellos ítems con una carga factorial mayor o igual a 0.40, los cuales, según Comrey (1973), serían adecuados para el análisis. De los nueve componentes anteriores se seleccionaron tres que explicarían el $18.28 \%$ de la varianza, debido a que los restantes contaban sólo con un ítem.

El primer componente de resiliencia hallado en el grupo se denominó: Actitudes y habilidades sociales de solución eficaz de problemas, el cual se re- fiere a comportamientos resilientes, tales como poner en práctica las propias competencias en situaciones difíciles, inventar formas de disminución de la tensión generada por un problema, darse cuenta de las cualidades que tienen las otras personas. El segundo componente resiliente hallado en los examinados fue el humor, que hace referencia a aspectos como la relajación después vivir una experiencia dolorosa o el uso la risa como alternativa para tranquilizarse en situaciones tensionantes. El tercer componente resiliente hallado se denominó conductas vitales positivas, con aspectos como tratar de mejorar los aspectos negativos de sí mismo. 
Tabla 1. Análisis factorial de los componentes de resiliencia en estudiantes universitarios

\begin{tabular}{|c|c|c|c|}
\hline \multicolumn{4}{|c|}{ Análisis Factorial } \\
\hline Nombre del componente & Ítem & Carga factorial & Expli. Varianza \\
\hline \multirow{24}{*}{$\begin{array}{l}\text { 1.Actitudes y habilidades } \\
\text { sociales de solución eficaz } \\
\text { de problemas }\end{array}$} & 9 & 0.440 & \multirow{24}{*}{$12.092 \%$} \\
\hline & 22 & 0.407 & \\
\hline & 25 & 0.491 & \\
\hline & 26 & 0.473 & \\
\hline & 28 & 0.488 & \\
\hline & 29 & 0.488 & \\
\hline & 40 & 0.525 & \\
\hline & 43 & 0.628 & \\
\hline & 46 & 0.558 & \\
\hline & 49 & 0.565 & \\
\hline & 50 & 0.463 & \\
\hline & 51 & 0.453 & \\
\hline & 53 & 0.526 & \\
\hline & 55 & 0.445 & \\
\hline & 58 & 0.549 & \\
\hline & 60 & 0.449 & \\
\hline & 64 & 0.454 & \\
\hline & 65 & 0.465 & \\
\hline & 66 & 0.604 & \\
\hline & 70 & 0.593 & \\
\hline & 75 & 0.572 & \\
\hline & 83 & 0.460 & \\
\hline & 88 & 0.512 & \\
\hline & 89 & 0.434 & \\
\hline \multirow{10}{*}{ 2. Humor } & 13 & 0.526 & \multirow{10}{*}{$4.799 \%$} \\
\hline & 23 & 0.546 & \\
\hline & 31 & 0.452 & \\
\hline & 32 & 0.414 & \\
\hline & 33 & 0.511 & \\
\hline & 37 & 0.405 & \\
\hline & 41 & 0.425 & \\
\hline & 68 & 0.466 & \\
\hline & 81 & 0.524 & \\
\hline & 86 & 0.530 & \\
\hline \multirow{3}{*}{ 3. Conductas vitales positivas } & 28 & 0.440 & \multirow{3}{*}{$1.389 \%$} \\
\hline & 61 & 0.483 & \\
\hline & 85 & 0.466 & \\
\hline Varianza explicada & & & $18,28 \%$ \\
\hline
\end{tabular}


Tabla 2. Correlaciones entre resiliencia y las variables: edad, estrato socioeconómico y número de hijos

\begin{tabular}{|l|r|r|}
\hline \multicolumn{3}{|c|}{ Spearman } \\
\hline Variables Socio demográficas & Resiliencia & \\
\hline Edad & Correlation Coefficient & -.254 \\
\hline & Sig. & .001 \\
\hline Estrato & $\mathrm{N}$ & 180 \\
\hline & Correlation Coefficient & .308 \\
\hline & Sig. & .000 \\
\hline Hijos & $\mathrm{N}$ & 180 \\
\hline & Correlation Coefficient & -.063 \\
\hline & Sig. & .404 \\
\hline
\end{tabular}

La Tabla 2 muestra los resultados de las correlaciones entre resiliencia y las variables sociodemográficas: edad, estrato y número de hijos, de la muestra de estudiantes universitarios objeto de estudio. La conclusión es que la correlación entre resiliencia y la edad muestra un coeficiente de correlación de Spearman $r s=-0.254$, lo cual corresponde a una correspondencia inversa o negativa débil, esto significa que a menor edad de los participantes, su capacidad de resiliencia sería mayor; sin embargo, estos valores no son suficientemente representativos. Respecto a la variable estrato, se observa un coeficiente de correlación de $r s=0.308$, lo cual corresponde a una correspondencia positiva débil, es decir, los participantes que pertenecen a un estrato alto serían más resilientes que los de menor nivel socioeconómico y viceversa; sin embargo, no es una correlación significativa. Finalmente respecto al número de hijos se obtiene un coeficiente de correlación de $r s=$ -0.063 , lo cual corresponde a una correspondencia inversa o negativa débil. Esto refiere que a menor número de hijos de los participantes su resiliencia sería mayor y viceversa, sin embargo, el valor no es significativo. En conclusión, las correlaciones halladas, si bien son directas, son a su vez débiles, por tanto, poco significativas.

Tabla 3. Diferencia de medias en la variable sociodemográfica: género

\begin{tabular}{|c|c|c|c|c|c|c|}
\hline & \multicolumn{6}{|c|}{ Prueba $t$ Student } \\
\hline & \multicolumn{6}{|c|}{ Género } \\
\hline & $t$ & $d f$ & Sig & $\begin{array}{l}\text { Diferencia de } \\
\text { medias }\end{array}$ & \multicolumn{2}{|c|}{$\begin{array}{c}95 \% \text { Intervalo de confianza de } \\
\text { la diferencia }\end{array}$} \\
\hline & & & & & Inferior & Superior \\
\hline Femenino & 14.542 & 84 & .000 & 81.494 & 70.35 & 92.64 \\
\hline \multirow[t]{5}{*}{ Total } & 63.845 & 84 & .000 & 383.294 & 371.36 & 395.23 \\
\hline & \multicolumn{6}{|c|}{ Prueba $t$ Student } \\
\hline & \multicolumn{6}{|c|}{ Género } \\
\hline & $t$ & $d f$ & Sig. & $\begin{array}{l}\text { Diferencia de } \\
\text { medias }\end{array}$ & \multicolumn{2}{|c|}{$\begin{array}{c}95 \% \text { Intervalo de confianza de } \\
\text { la diferencia }\end{array}$} \\
\hline & & & & & Inferior & Superior \\
\hline Masculino & 18.834 & 95 & .000 & 98.396 & 88.02 & 108.77 \\
\hline Total & 65.811 & 95 & .000 & 400.042 & 387.97 & 412.11 \\
\hline
\end{tabular}


La Tabla 3 muestra las diferencias de medias entre el género, estado civil y el estado de convivencia familiar. Se hallaron valores $t$ superiores a los esperados con $p<0.01$, confirmando diferencias de género en la capacidad de resiliencia de los estudiantes examinados. En el caso femenino, una $t=14.542$; y en el masculino, una $t=18.834$.
La Tabla 4 muestra los mismos resultados al comparar la capacidad de resiliencia de los estudiantes que viven con su familia con respecto a los que no, siendo mayor en los primeros. Los valores de $t$, con $p<0.01$, fueron de $t=21.838$ (para quienes viven con su familia) y $t=8.489$ (para quienes no viven con la familia).

Tabla 4. Diferencia de medias en la variable sociodemográfica: estado de convivencia familiar

\begin{tabular}{|c|c|c|c|c|c|c|}
\hline & \multicolumn{6}{|c|}{ Prueba $t$ de Student } \\
\hline & \multicolumn{6}{|c|}{ Estado de convivencia familiar } \\
\hline & $t$ & $d f$ & Sig. & $\begin{array}{c}\text { Diferencia de } \\
\text { medias }\end{array}$ & \multicolumn{2}{|c|}{$\begin{array}{l}\text { 95\% Intervalo de confianza } \\
\text { de la diferencia }\end{array}$} \\
\hline & & & & & Inferior & Superior \\
\hline Familia SI & 21.838 & 147 & .000 & 93.155 & 84.73 & 101.59 \\
\hline \multirow[t]{5}{*}{ TOTAL } & 82.314 & 147 & .000 & 394.953 & 385.47 & 404.43 \\
\hline & \multicolumn{6}{|c|}{ Prueba $t$ de Student } \\
\hline & \multicolumn{6}{|c|}{ Estado de convivencia familiar } \\
\hline & $t$ & $d f$ & Sig. & \begin{tabular}{|c|c|}
$\begin{array}{c}\text { Diferencia de } \\
\text { medias }\end{array}$ \\
\end{tabular} & \multicolumn{2}{|c|}{$\begin{array}{c}\text { 95\% Intervalo de confianza } \\
\text { de la diferencia }\end{array}$} \\
\hline & & & & & Inferior & Superior \\
\hline Familia NO & 8.489 & 31 & .000 & 78.219 & 59.43 & 97.01 \\
\hline TOTAL & 37.847 & 31 & .000 & 379.250 & 358.81 & 399.69 \\
\hline
\end{tabular}

Tabla 5. Diferencia de medias en la variable sociodemográfica: estado civil

\begin{tabular}{|c|c|c|c|c|c|c|}
\hline & \multicolumn{6}{|c|}{ Prueba $t$ de Student } \\
\hline & \multicolumn{6}{|c|}{ Estado Civil } \\
\hline & \multirow[t]{2}{*}{$t$} & \multirow[t]{2}{*}{$d f$} & \multirow[t]{2}{*}{ Sig. } & \multirow[t]{2}{*}{$\begin{array}{l}\text { Diferencia de } \\
\text { medias }\end{array}$} & \multicolumn{2}{|c|}{$\begin{array}{l}\text { 95\% Intervalo de confianza } \\
\text { de la diferencia }\end{array}$} \\
\hline & & & & & Inferior & Superior \\
\hline Solteros & 23.157 & 164 & .000 & 92.867 & 84.95 & 100.79 \\
\hline \multirow[t]{5}{*}{ TOTAL } & 87.065 & 164 & .000 & 395.036 & 386.08 & 404.00 \\
\hline & \multicolumn{6}{|c|}{ Prueba $t$ de Student } \\
\hline & \multicolumn{6}{|c|}{ Estado Civil } \\
\hline & $t$ & $d f$ & Sig. & $\begin{array}{l}\text { Diferencia de } \\
\text { medias }\end{array}$ & \multicolumn{2}{|c|}{$\begin{array}{c}95 \% \text { Intervalo de confianza } \\
\text { de la diferencia }\end{array}$} \\
\hline & & & & & Inferior & Superior \\
\hline Casados & 3.333 & 8 & .010 & 50.778 & 15.65 & 85.91 \\
\hline \multirow[t]{5}{*}{ TOTAL } & 27.884 & 8 & .000 & 347.000 & 318.30 & 375.70 \\
\hline & \multicolumn{6}{|c|}{ Prueba $t$ de Student } \\
\hline & \multicolumn{6}{|c|}{ Estado Civil } \\
\hline & $t$ & $d f$ & Sig. & $\begin{array}{l}\text { Diferencia de } \\
\text { medias }\end{array}$ & \multicolumn{2}{|c|}{$\begin{array}{l}\text { 95\% Intervalo de confianza } \\
\text { de la diferencia }\end{array}$} \\
\hline & & & & & Inferior & Superior \\
\hline Unión Libre & 3.396 & 5 & .019 & 85.000 & 20.67 & 149.33 \\
\hline TOTAL & 15.442 & 5 & .000 & 380.833 & 317.44 & 444.23 \\
\hline
\end{tabular}


En lo referente a la variable estado civil, se encontró mayor resiliencia en solteros ( $t=23.157$, con $p<0.01)$. En los demás estados civiles no se hallaron diferencias significativas (unión libre, $t=3.396$ con $p<0.01$ ), tal como se ve en la Tabla 5.

En la Tabla 6 se muestran las correlaciones entre Resiliencia y Rendimiento Académico (Promedio acumulado, Promedio actual, Semestres no aprobados, Semestres aplazados) en la muestra estudiada. Se halló una correlación negativa débil entre Resiliencia y Promedio Acumulado ( $r s=-0.090)$. Respecto a la variable Promedio actual y Resiliencia, se halló igualmente una correlación negativa débil $(r s=-0.157)$. El mismo tipo de correspondencia se encontró entre la capacidad de Resiliencia y los Semestres perdidos ( $r s=$ -0.029). Asimismo, se halló entre la Resiliencia y los Semestres aplazados $(r s=-0.142)$.También se halló lo mismo entre Resiliencia y Asignaturas pendientes ( $r s=$ 0.192) y entre Resiliencia y Asignaturas no aprobadas $(r s=0.105)$.

Tabla 6. Resultados de las correlaciones entre resiliencia y rendimiento académico

\begin{tabular}{|c|c|c|}
\hline \multirow{2}{*}{ Rendimiento académico } & \multicolumn{2}{|c|}{ Muestra } \\
\hline & \multicolumn{2}{|c|}{ Resiliencia } \\
\hline \multirow[t]{3}{*}{ Promedio Acumulado } & Correlation Coefficient & -.090 \\
\hline & Sig. & .231 \\
\hline & $N$ & 180 \\
\hline \multirow[t]{3}{*}{ Promedio Actual } & Correlation Coefficient & -.157 \\
\hline & Sig. & .036 \\
\hline & $\mathrm{N}$ & 180 \\
\hline \multirow[t]{3}{*}{ Semestres Perdidos } & Correlation Coefficient & -.029 \\
\hline & Sig. & .701 \\
\hline & $N$ & 180 \\
\hline \multirow[t]{3}{*}{ Semestres Aplazados } & Correlation Coefficient & -.142 \\
\hline & Sig. & .057 \\
\hline & $N$ & 180 \\
\hline \multirow[t]{3}{*}{ Asignaturas Pendientes } & Correlation Coefficient & .192 \\
\hline & Sig. & .010 \\
\hline & $N$ & 180 \\
\hline \multirow[t]{3}{*}{ Asignaturas No Aprobadas } & Correlation Coefficient & .105 \\
\hline & Sig. & .161 \\
\hline & $N$ & 180 \\
\hline
\end{tabular}

\section{DISCUSIÓN}

Los hallazgos del estudio muestran tres factores resilientes en el grupo evaluado, a saber: Humor, capacidades de solución de problemas y conductas vitales positivas. Si bien, estudios como el de Peralta, Ramírez \& Castaño (2006) muestran un espectro de factores más amplio, el presente estudio mantiene coincidencias con él en los factores encontrados. Sin embargo, otras dimensiones de la resiliencia no fueron identificadas en este grupo y son de alta relevancia, por ejemplo, la capacidad de introspección, necesaria para reflexionar sobre la propia experiencia y organizar el mundo interior afectivo para hacerlo más sólido ante las demandas de adaptación del entorno. Tampoco se encontraron otras como la independencia, la moralidad y el pensamiento crítico. Esto significa que, en general, la muestra evaluada de estudiantes tiende a mantener una orientación más hacia el exterior, esto es, hacia resolver los problemas de manera práctica confiando en sí mismo y manteniendo una actitud proactiva pero sin reflexionar demasiado acerca de sí mismo y de la realidad 
que los desafía. Esto puede resultar problemático en cierto sentido, ya que la resiliencia está atada a la independencia, la cual resulta posible a través del mayor conocimiento de si y de las propias potencialidades y limitaciones, de tal manera que el individuo se asume responsable frente a sí mismo y a lo que puede controlar de su vida, dejando para la solicitud de ayuda a los demás aquello que en justicia escapa a su propio potencial de realización y dominio. Con respecto a la hipótesis, los hallazgos confirman lo conjeturado: en efecto se obtuvieron componentes de humor e iniciativa. Esto hace que el grupo examinado se ubique en el promedio general de los componentes mínimos que cabría esperar en la resiliencia, por supuesto, como se ha anotado, con carencias importantes.

Con respecto a las correlaciones entre la resiliencia y la edad, el estrato socioeconómico y el rendimiento académico, los hallazgos mostraron correlaciones negativas y débiles, lo cual indica que, al parecer, la resiliencia no se relaciona con que las personas, en este caso los estudiantes, tengan más edad, o con que su condición para el acceso a adecuadas condiciones de calidad de vida sean mayores; tampoco con que resulta en un mayor rendimiento académico, lo que indica que la edad no hace más o menos resiliente a una persona, es decir, puede haber adultos muy poco resilientes o jóvenes muy resilientes. Por otra parte, que el ambiente y sus condiciones pueden operar con independencia de la capacidad resiliente de un sujeto al no condicionarla, esto es, personas con calidad de vida deficiente o carente podrían ser más resilientes que aquellos con condiciones de vida cómodas. En general, estos hallazgos son contrarios a lo hallado por Gargallo, Pérez et al. (2003), quienes encontraron correlaciones positivas entre actitudes positivas y rendimiento académico, al igual que el estudio de Escurra et al. (2004), donde los hallazgos indicaban que el auto concepto y las metas influyen de forma positiva en el rendimiento. Con todo, la hipótesis de trabajo apuntaba a corroborar estos mismos antecedentes, lo cual no se halló.

Finalmente, los resultados obtenidos en las correlaciones de la resiliencia y la condición de estar casado o soltero contradicen la hipótesis de partida de este estudio, puesto que lo hallado señala esto último, en otras palabras: estar casado o soltero no parece asociarse con tener mayor o menor grado de resiliencia, en este caso en situaciones del entorno educativo universitario. Con respecto a la variable género del presente estudio, en ambos casos (masculino y femenino), existe una diferencia significativa en las muestras con respecto a su capacidad de resiliencia siendo mayor en las mujeres estudiadas. En concreto, podría afirmarse que las mujeres parecen ser más resilientes que los hombres estudiantes universitarios. Esto a su vez confirma lo planteado en la hipótesis de trabajo con respecto al género. Podría suponerse que los recursos de adaptación a situaciones estresantes académicas en las mujeres son más efectivos en cuanto que se interesan menos por competir y lograr, lo cual parece ser más destacado en el grupo masculino que tiende a buscar más reconocimiento social y aprobación a través del desempeño eficiente en una actividad que lo destaca socialmente y lo reafirma personalmente. En cuanto el estado de convivencia familiar, se observa un resultado interesante puesto que aparecen niveles de resiliencia superiores en quienes se separan de sus familias con motivo de atender sus asuntos educativos; además, está en la misma línea de los hallazgos de otros estudios (DiGresia, Porto \& Ripiani, 2001), quienes concluyeron que si el alumno cambia de residencia ese cambio tiene efectos positivos sobre el rendimiento, frente a lo cual los autores sugieren que la más rápida integración al ambiente universitario supera el desarraigo y la separación de la familia.

\section{CONCLUSIONES}

En resumen podría afirmarse que según lo hallado en el presente estudio, la resiliencia en estudiantes universitarios no parece estar asociada con la edad de los mismos, con su rendimiento académico, el estrato socioeconómico del que proceden, su estado civil, su número de hijos, pero si parece existir una diferencia con respecto al género, pues las mujeres parecieran mostrarse más resilientes que los hombres ante las exigencias del proceso educativo universitario, también parece existir diferencia en cuanto a que quienes se independizan de sus familias parecen responder mejor a sus estudios. 


\section{REFERENCIAS}

Bermúdez, S., Durán, M., Escobar, C., Morales, A., Monroy, S. \& Ramírez, A., (2006). Evaluación de la relación entre rendimiento académico y estrés en estudiantes de medicina. MEDUNAB, 9, 198-205.

Bradley, R., Whitesid, L., Mudfrom, D., Casey, P., Keller, K. \& Pope, S. (1994). Early indicators of resilience and their relation to experiences in the home environments of low birth weight, premature children living in poverty. Child Development, 65(2), 346-360.

Bragagnolo, G., Rinarudo, A., Cravero, N., Fomía, S., Martínez, G. \& Vergara, S. (2005). Optimismo, esperanza, autoestima y depresión en estudiantes de Psicología. Informe de investigación. Buenos Aires: Universidad Nacional de Rosario.

Di Gresia, L., Porto, A. \& Ripani, L. (2002). Rendimiento de los estudiantes de las universidades públicas argentinas. Universidad Nacional de la Plata. Facultad de Ciencias Económicas 2002. Documento de Trabajo No. 45, 1-40.

Escurra, L., Delgado, A., Guevara, G., Torres, M., Quezada, R., Morocho, J. (2005). Relación entre el autoconcepto de las competencias, las metas académicas y el rendimiento en alumnos universitarios de la Universidad de Lima. Revista IIPSI, 8(1), 87-106.

Gargallo, B., Pérez, C., Serra, B., Sánchez, F. \& Ross, I. (2007). Actitudes ante el aprendizaje y rendimiento académico en los estudiantes universitarios. Revista Iberoamericana de Educación, 1(42), 2-11.

Garmezy, N. (1994). Reflections and commentary on risk, resilience and development. En: Haggerty, R., Sherral, N., Gamezy, N. \& Rutter, M. (Eds.). Stress, risk and resilience in children and adolescents: Processes, mechanisms and interventions. Cambridge University Press.

Peralta, S., Ramírez, A. \& Castaño, H. (2006). Factores resilientes asociados al rendimiento académico en estudiantes pertenecientes a la Universidad de Sucre (Colombia). Psicología desde el Caribe, 17, 196-219.

Sammeroff, A. \& Seifer, R. (1992). Early contributors to developmental risk. En: Rolf, J., Masten, A., Cicchetti, D., Neuechterlein, K \& Weintraub, S. (Eds.). Risk and protective factors in the development of psychopatho$\log y$. Cambridge University Press.

Solórzano, M. \& Ramos, N. (2006). Rendimiento académico y estrés académico de los estudiantes de la
E.A.P. de enfermería de la Universidad Peruana Unión (Semestre I-2006). Rev. De Ciencias de la Salud, 1(1), 34-38.

Werner, E. (1989). High risk children in young adulthood: A longitudinal study from birth to 32 years. American Journal of Ortho Psyquiatry, 59(1), 72-81.

Wolín, S. \& Wolín, B. (1993). The resilient self: How survivors of troubled families rise above Diversity. New York: Villard Books.
Fecha de recepción: Abril 2010 Fecha de publicación: Septiembre 2010 